\title{
Qualitative Identification and Semi-Quantitative Comparison of Sucrose in Maca (Lepidium meyenii) by Infrared Spectrum Analysis
}

\author{
Xianxue Wu ${ }^{1,2^{*}}$, Wenjing Chen ${ }^{1}$, Liangxing Li ${ }^{1}$, Binqiang Xu${ }^{1}$, Yuehai Guo ${ }^{1}$ \\ ${ }^{1}$ College of Chemistry Biology and Environment, Yuxi Normal University, Yuxi, China \\ ${ }^{2}$ Academic Affairs Office, Yuxi Normal University, Yuxi, China \\ Email: xxw@yxnu.net
}

How to cite this paper: $\mathrm{Wu}, \mathrm{X} . \mathrm{X}$., Chen, W.J., Li, L.X., Xu, B.Q. and Guo, Y.H. (2018) Qualitative Identification and Semi-Quantitative Comparison of Sucrose in Maca (Lepidium meyenii) by Infrared Spectrum Analysis. American Journal of Analytical Chemistry, 9, 322-329.

https://doi.org/10.4236/ajac.2018.96025

Received: April 18, 2018

Accepted: June 18, 2018

Published: June 21, 2018

Copyright $\odot 2018$ by authors and Scientific Research Publishing Inc. This work is licensed under the Creative Commons Attribution-NonCommercial International License (CC BY-NC 4.0). http://creativecommons.org/licenses/by-nc/4.0/ (c) (i) \& Open Access

\begin{abstract}
Objective: In order to establish a method for qualitative identification and semi-quantitative comparison of sucrose in Macas by infrared spectral analysis. Methods: Six Maca samples with three color types and from two producing areas in Yunnan province (China) were collected. Fourier transform infrared spectroscopy (FTIR) of raw Maca, ethyl acetate extracts (EtOAc-E), alcohol extracts (EtOH-E) and extracted residues were recorded selectively. Comparative analysis and analysis-through-separation strategies were used to explore the FTIR analytical method for sucrose in Maca. Results: Sucrose was proved to be a component in the Lijiang purple Maca (LPM) sample by comparative analysis of FTIR and second derivative infrared spectroscopy (SDIR) with sucrose as a reference substance. Relative higher content of sucrose might be responsible for the distinctive absorption character of FTIR from LPM. With the aid of comparative analysis and analysis-through-separation strategies, conclusions could be drawn that all of the six Maca samples in this research contained some sucrose more or less. Furthermore, relative content of sucrose in the LM was higher than those in HM in general. Conclusion: An infrared spectrum analysis method for qualitative identification and semi-quantitative comparison of sucrose in different Maca samples was developed. Compared with other analytical methods, the developed method shows unparalleled charm for its inherent merits of convenient, fast, low cost, visual, efficient et al.
\end{abstract}

\section{Keywords}

Maca (Lepidium meyenii), Sucrose, FTIR, SDIR, Comparative Analysis 


\section{Introduction}

Maca, Lepidium meyenii (Brassicaceae), is an annual herbaceous plant native to the high plateaus of the Peruvian central Andes [1]. Its roots have high nutritional and medicinal value, which have long been used as food and ethnic medicine. Maca has many pharmacological activities such as anti-fatigue, enhancing immunity, increasing fertility, anti-inflammation, anti-cancer, anti-oxidation, anti-virus, lowering blood pressure and treating menopausal syndrome [2]. Main primary metabolites of Maca were protein, starch, dietary fiber, fatty acid, etc. And secondary metabolites of Maca were Macamides, alkaloid, glucosinolate, sterol and so on [3] [4] [5] [6] [7]. Few reports about small molecule saccharides in Maca had been found [8] [9].

FTIR plays an important role in the structure analysis of compound functional groups. Taking advantage of merits including direct, simple, fast and nondestructive, it also shows unique guiding role and unparalleled vitality in the analysis of complex mixture system [10] [11]. The FTIR of a complex mixture sample could be regarded as the nonlinear superposition of the infrared spectra of all compounds in the mixture. It has very strong fingerprint characteristics, just like the intrinsic fingerprint characteristics of the FTIR of a compound. Although heavy overlaps of absorption peaks might bring difficulties for analysis of complex mixture, the intact and original material information hidden behind those peaks was worth digging. SDIR, obtained by the second derivative processing of raw FTIR, was often used in FTIR analysis for the sake of higher apparent resolution. Based on Bill's law, infrared spectral analysis can also be used for qualitative and even quantitative analysis, just as other spectral analysis methods working. Based on this understanding, we have been focused on the infrared spectral analysis of complex mixture including traditional Chinese medicine, tobacco, tea, oil, in recent years [12] [13] [14] [15] [16].

We had noticed that the FTIR of Lijiang purple Maca (LPM) shown distinctive absorption characteristic compared to those of others in our research work, and that was the work we are going to do. As part of our continuing research work, we look forward to disclose the material reason leads to the distinctive peaks.

\section{Experiment}

\subsection{Apparatus}

Frontier FTIR spectrometer (Perkin-Elmer) equipped with a deuterated triglycine sulfate (DTGS) detector was used. A 10 tons tablet press (Specac) was used for preparation of sample disk for transmission FTIR.

\subsection{Samples and Reagents}

Six Maca (Lepidium meyenii) samples, including color types of purple, black and yellow from Lijiang and Huize (Yunnan, China) producing areas respectively, were collected from local market in 2016. 
$\mathrm{KBr}$ (specpure) were purchased from Zigong Sanchuan Chemical Reagent Co., Ltd. (Sichuan, China). All other reagents, including sucrose, ethanol and ethyl acetate, were all analytical pure reagents purchased from Chinese chemical reagent companies.

All FTIR spectra were recorded from an accumulation of 32 scans, and 0.2 $\mathrm{cm} / \mathrm{s}$ of optical path difference (OPD) speed in the range of $4000-400 \mathrm{~cm}^{-1}$ with a resolution of $4 \mathrm{~cm}^{-1}$. The interferences of $\mathrm{H}_{2} \mathrm{O}$ and $\mathrm{CO}_{2}$ were subtracted when scanning.

\subsection{Procedure}

All six Maca samples were smashed to 100 mesh after drying at $50^{\circ} \mathrm{C}$ and reserved for test. $5.0 \mathrm{~g}$ Huize Maca (HM) powder was soaked in $50 \mathrm{~mL}$ extraction solvent (EtOAc and $75 \% \mathrm{EtOH}$ ) at room temperature for a week. Extract and extracted residue was prepared for IR test by centrifugal separation and rotary evaporation operations successively. Each pre-prepared sample (about 1 - $2 \mathrm{mg}$ ) was blended with $\mathrm{KBr}(100 \mathrm{mg})$, grounded into powder (200 mesh), and then pressed into a tablet for recording the spectra.

\subsection{Data Processing}

The raw FTIR data was processed with Perkin-Elmer infrared spectrum software (Spectrum 10.0). The SDIR were obtained after normalization and Savitzky-Golay polynomial fitting (13-point smoothing) of the original FTIR after baseline correction. All functions mentioned above were integrated in Spectrum 10.0 and could be realized by tapping the keyboard.

\section{Results and Discussion}

\subsection{Identification of Sucrose in LPM by Comparative Analysis of FTIR and SDIR Spectra}

Overlap diagram of 30 FTIR spectra collected from 6 kinds of Maca samples was prepared (Figure 1). As was shown in Figure 1, the 5 paralleled absorption spectra of FTIR from LPM was significantly different from those of rest Maca samples especially in the vicinity of 3330 and $1050 \mathrm{~cm}^{-1}$. It showed stronger and sharper absorption peaks at 3562, 3388, 3338, 1069, 1053, 1014, 991 and 921 $\mathrm{cm}^{-1}$. Judging by elementary theory of FTIR, such absorption characteristics should always be attributed to some small molecular saccharide. In order to discover the chemical reason of distinctive absorption peaks such as $3562 \mathrm{~cm}^{-1}$, we had searched various small molecule saccharides from FTIR database extensively. Surprisingly, all foresaid characteristic peaks could be found in FTIR of sucrose searched from database. However, nearly none report mentioned the existence of sucrose in Maca except for two papers reported lately [1] [4]. Therefore, FTIR of sucrose was collected and used as reference spectrum for comparative analysis with that of LPM (Figure 2).

It was easy to find that FTIR of LPM had nearly all characteristic absorption 


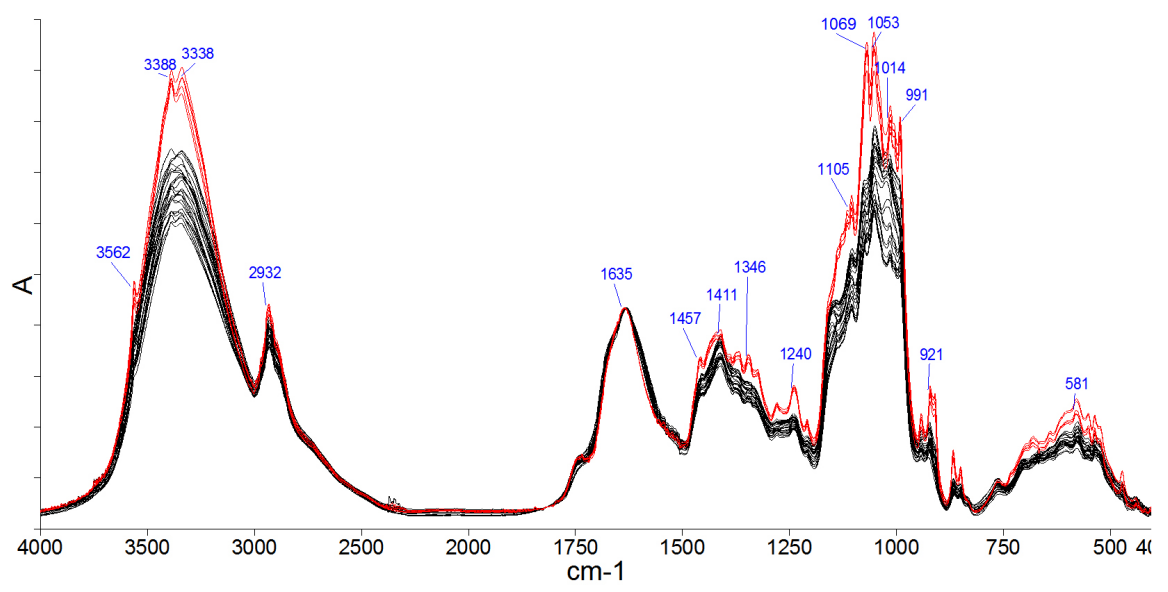

Figure 1. Stacked plot of FTIR spectra from LPM (Red) and rest Macasamples (Black) $\left(4000-400 \mathrm{~cm}^{-1}\right)$.

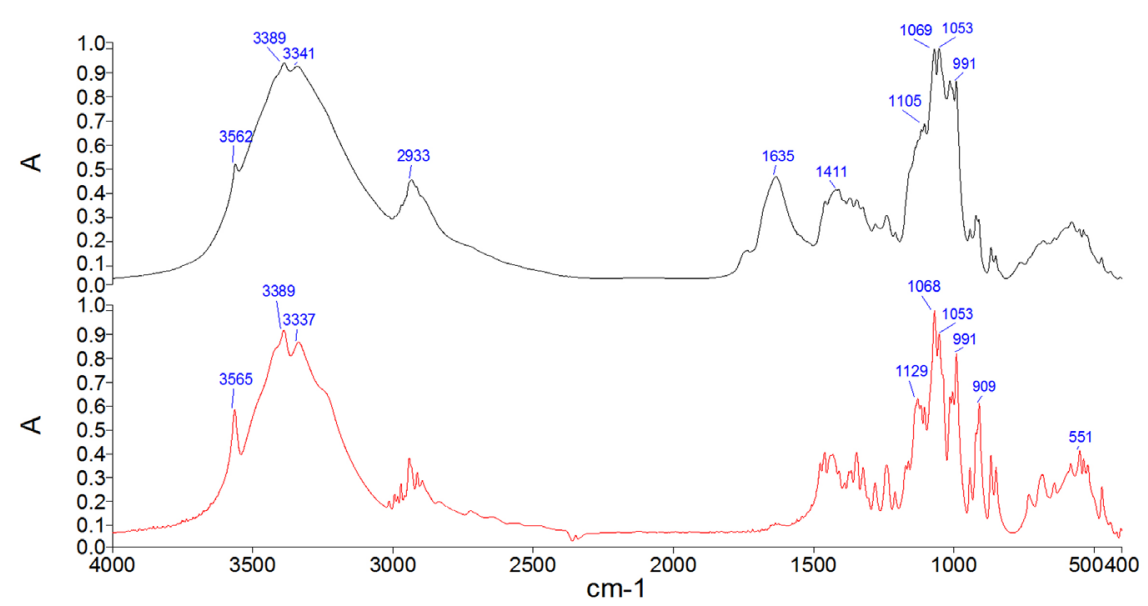

Figure 2. Separated plot of FTIR of LPM (up) and sucrose $\left(4000-400 \mathrm{~cm}^{-1}\right)$.

peaks of sucrose such as3565 (3562), 3389, 3337 (3341), 1069, 1053 and $991 \mathrm{~cm}^{-1}$ in Figure 1. In addition, it was not difficult to see that both shape and position of those peaks was similar to that of sucrose, especially in the ranges of 3700 $2800 \mathrm{~cm}^{-1}$ and $1200-900 \mathrm{~cm}^{-1}$. As result, it is hard to deny some amount of sucrose existed in the LPM sample.

In view of higher apparent resolution of the SDIR, second derivative processing of FTIR of both LPM and sucrose was done. And two representative sections were chosen for analysis (Figure 3).

As was shown in Figure 3, more characteristic peaks of sucrose could be found in SDIR of LPM visually. Specifically, they were 3365 (3364), 3388 (3390), 3014, 2995, 2972, 2944, 2914, 1460 (1462), 1323, 1280, 1209, 1162 (1161), 1139, 1105, 1068, 1053 (1054), 1037, 1015, 991 (990), 943, 922, 909, 868, 849 (850) $\mathrm{cm}^{-1}$, etc. Accordingly, it was unambiguous that the LPM sample does contain some sucrose component.

Up to now, conclusion could be drawn undoubtedly that sucrose was existed in the LPM by comparative analysis of FTIR and SDIR between LPM and 


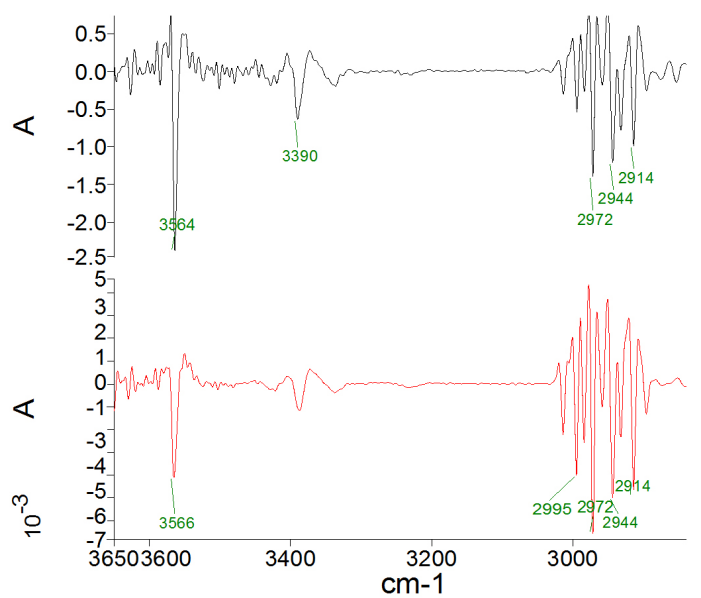

(a)

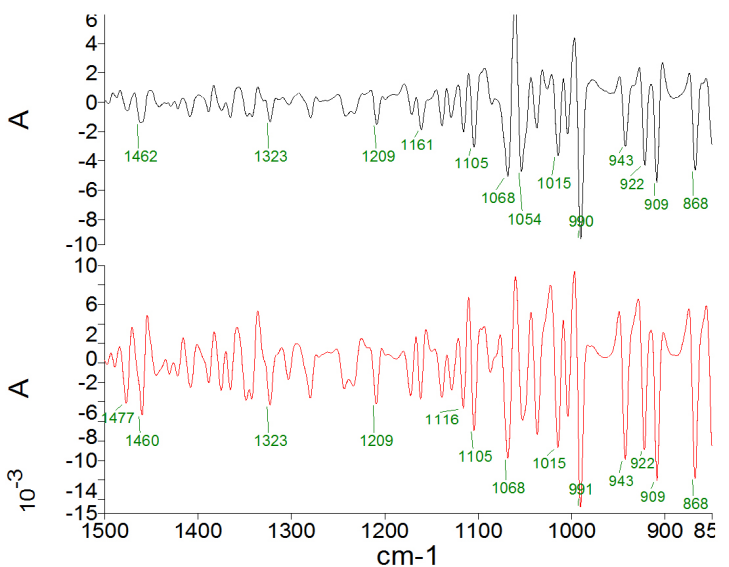

(b)

Figure 3. Separated plot of SDIR of LPM (up) and sucrose: Left: $3650-2870 \mathrm{~cm}^{-1}$; Right: $1500-840 \mathrm{~cm}^{-1}$.

sucrose. The conclusion was consistent with literature [8] [9], in which it was claimed that sucrose was extracted from Maca.

\subsection{Identification of Sucrose in Six Maca Samples by Comparative Analysis of SDIR}

To understand whether sucrose is a common ingredient of Maca, similar comparative analysis was carried out among SDIR spectra from six Maca samples as followed.

For both convictive conclusion and legible graph, only three necessary wavenumber ranges, including $3580-3545,3000-2900$ and $1000-900 \mathrm{~cm}^{-1}$, were chosen for analysis (Figure 4).

Whether from the view of characteristic peaks or trend line of each spectrum, it could be "see" that 3 Lijiang Maca (LM, red spectral line) was highly consistent with the blue spectral line of sucrose, which mean that the existence of sucrose in the LM samples. However, characteristic peaks of sucrose such as 3654, 2996, 2972, 2944, 2914, 990, 943, 922 and $909 \mathrm{~cm}^{-1}$ in the spectra of three Huize Maca 


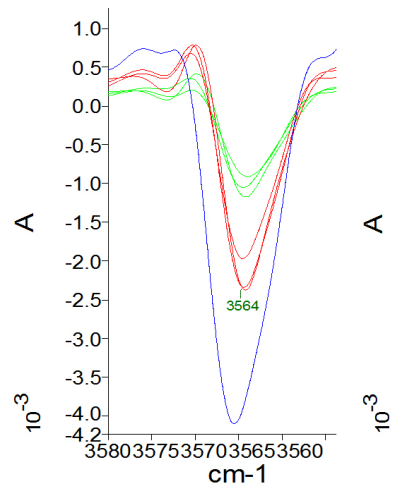

(a)

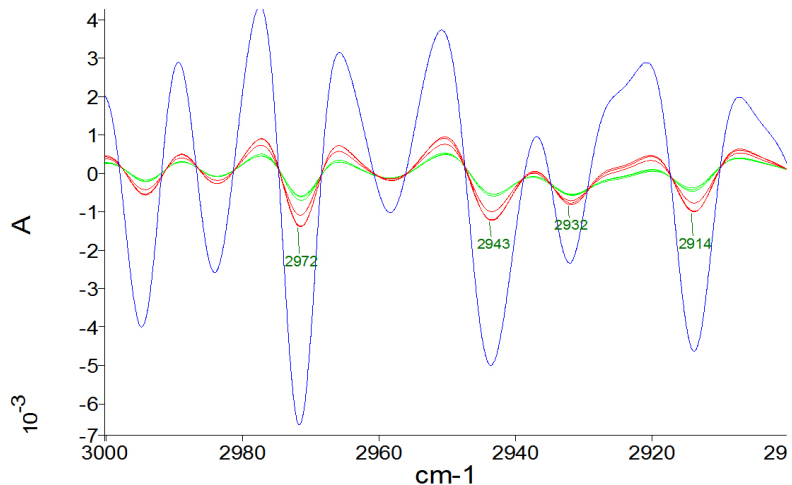

(b)

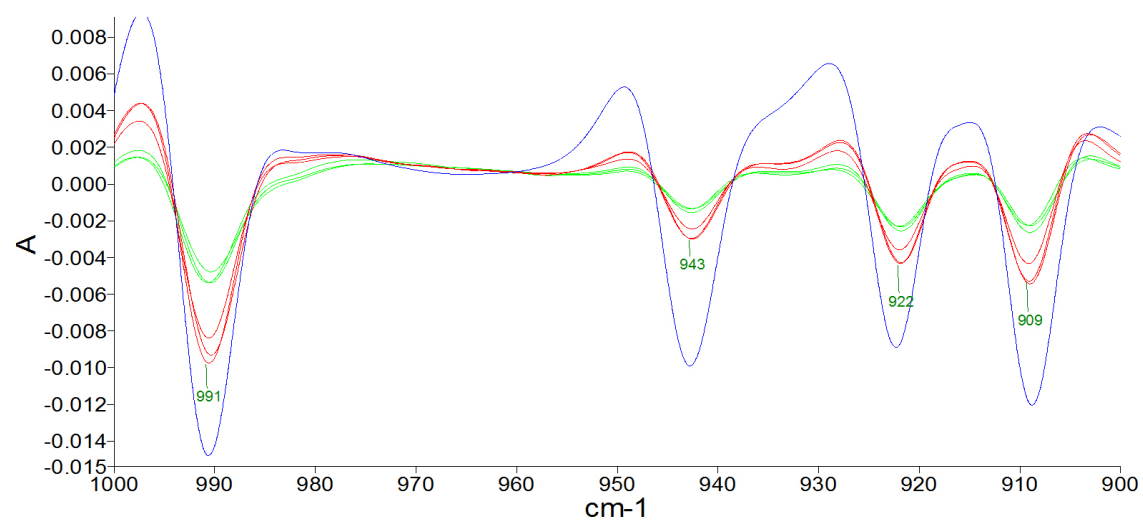

(c)

Figure 4. Stacked graphs of SDIR from 6 Maca and sucrose (LM: Red; HM: Green; sucrose: blue).

(HM) samples were too weak to use for identification of sucrose certainly. In fact, only SDIR of Huize purple Maca (HPM) had enough intensive characteristic peaks to identified sucrose unambiguously.

\subsection{Identification of Sucrose in Three HM Samples by Analysis-Through-Separation Strategy}

Analysis-through-separation is an efficient analytical strategy in infrared spectrum analysis of complex mixture system. As far as this paper concerned, sucrose is insoluble in EtOAc but partial soluble in $75 \% \mathrm{EtOH}$ according to similar dissolve mutually theory. Therefore, it could be deduced that sucrose would be enriched in EtOAc extracted residue, but be lacked in EtOH (75\%) extracted residue. What's more, these changes and differences would be recorded and displayed in SDIR. Hence, extraction experiments were designed and done.

As expected, sucrose characteristic peak sat 990, 943, 922 and $909 \mathrm{~cm}^{-1}$ from EtOAc extracted residues (red) became intensive compared with corresponding peaks of HM in Figure 4 (Figure 5). But, those peaks from EtOH (75\%) extracted residues got rather smooth and became almost invisible.

The results indicated that sucrose was still a component in HM samples but just a minor component when compared with LM in general. 


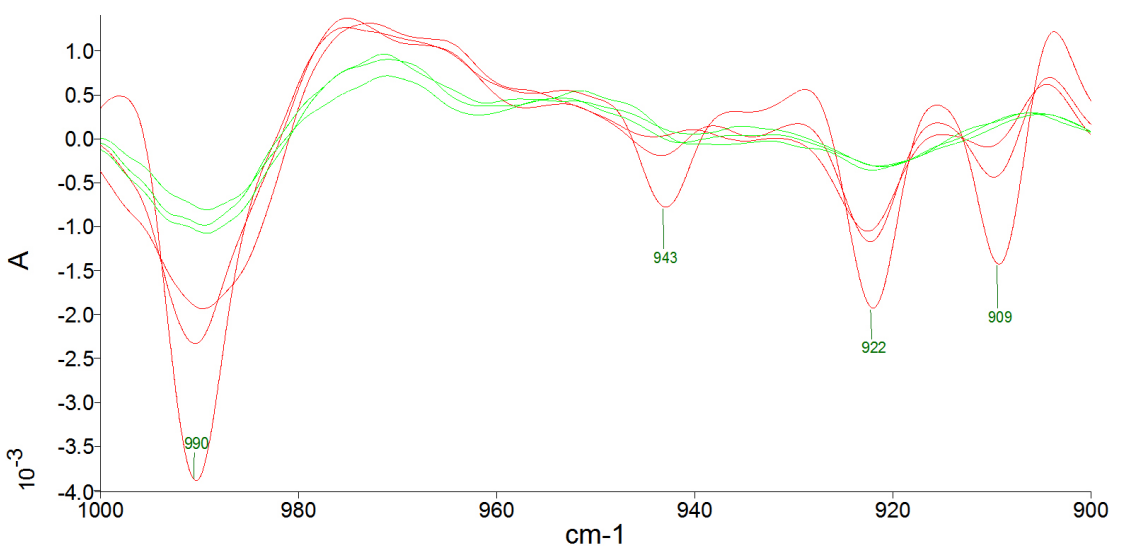

Figure 5. SDIR of EtOAc (Red) and EtOH (Green) extracted residue from HM (1000 $\left.900 \mathrm{~cm}^{-1}\right)$.

\section{Conclusions}

Through comparative analysis of FTIR and SDIR, it was confirmed that sucrose was existed in the LPM sample. Relatively high content of sucrose might be responsible for the distinctive absorption character of FTIR from LPM. With the aid of analytical strategies including comparative analysis and analysis-through-separation, conclusions could be drawn from the perspective of FTIR analysis that all six Maca samples in this research contained some sucrose more or less. Furthermore, relative content of sucrose in the LM was higher than those in HM in general.

An infrared spectrum analysis method for qualitative identification and comparison of sucrose in different Maca samples was developed. Compared with other analytical methods, the developed method shows unparalleled charm for its inherent merits of convenient, fast, low cost, visual, efficient et al.

\section{Acknowledgements}

This work was sponsored by National Natural Science Foundation of China (31560483) and Science Foundation of Yunnan province education department (2017ZDX135).

\section{References}

[1] Eliana, E., Antonella, H., Waltraud, K., et al. (2015) Bioactive Maca (Lepidium meyenii) Alkamides Are a Result of Traditional Andean Postharvest Drying Practices. Phytochemistry, 116, 138-148.

https://doi.org/10.1016/j.phytochem.2015.02.030

[2] Muhammad, I., Zhao, J.P., Dunbar, D.C., et al. (2002) Constituents of Lepidium meyenii "Maca". Phytochemistry, 59, 105-110. https://doi.org/10.1016/S0031-9422(01)00395-8

[3] Xu, M., Xu, L., Song, H., et al. (2015) Research Advances on Maca. Journal of Food Safety and Quality, 7, 2775-2782.

[4] Celine, C., Diego, D.G., Bharathi, A., et al. (2010) Influence of Colour Type and Previous Cultivation on Secondary Metabolites in Hypocotyls and Leaves of Maca 
(Lepidium meyenii Walp). Journal of the Science of Food and Agriculture, 90, 861-869.

[5] Tang, W.M., Jin, L., Xie, L.H., et al. (2017) Structural Characterization and Antifatigue Effect in Vivo of Maca (Lepidium meyenii Walp) Polysaccharide. Journal of Food Science, 82, 757-764. https://doi.org/10.1111/1750-3841.13619

[6] Pan, Y., Zhang, J., Li, H., et al. (2016) Characteristic Fingerprinting Based on Macamides for Discrimination of Maca (Lepidium meyenii) by LC/MS/MS and Multivariate Statistical Analysis. Journal of the Science of Food and Agriculture, 96, 4475-4483. https://doi.org/10.1002/jsfa.7660

[7] Wang, Y.L., Wang, Y.C., Brian, M.N., et al. (2007) Maca: An Andean Crop with Multi-Pharmacological Functions. Food Research International, 40, 783-792. https://doi.org/10.1016/j.foodres.2007.02.005

[8] Zang, Z., Lei, Z., Zhu, H.Y., et al. (2014) Chemical Constituents from Maca (Lepidium meyenii). Yunnan Chemical Technology, 4, 37-39.

[9] Du, P., Yang, M., Zhu, Y.Q., et al. (2016) Analysis of the Functional Constituent in Maca (Lepidium meyenii) from Yunnan. Science and Technology in Food Chemistry, 16, 71-75.

[10] Sun, S.Q., Chen, J.B., Zhou, Q., et al. (2010) Application of Mid-Infrared Spectroscopy in the Quality Control of Traditional Chinese Medicines. Planta Medica, 76, 1987-1996. https://doi.org/10.1055/s-0030-1250520

[11] Sun, S.Q., Chen, J.B. and Zhou, Q. (2013) ATC 009 Infrared Spectral Analysis Technology. China Quality Inspection Press, Beijing.

[12] Huang, H.M., Li, L.X., Chen, W.J., Wu, Y.Y. and Wu, X.X. (2016) Tracking Analysis of Frying Soybean Oil by ATR-FTIR. Chemical Research and Application, 8, 1169-1173.

[13] Ma, J., Wu, X.X., Tai, X., Xu, L.X., Zhu, J.L., Qin, Y., Zhou, Q. and Sun, S.Q. (2014) Tracking Analysis of Three Extraction Processes of Arenaria polytrichoides by Fourier Transform Infrared Spectrocopy. Spectroscopy and Spectral Analysis, 10, 2833-2838.

[14] Wu, X.X., Xu, C.H., Li, M., Sun, S.Q., Li, J.M. and Dong, W. (2014) Analysis and Identification of Two Reconstituted Tobacco Sheets by Three-Level Infrared Spectroscopy. Journal of Molecular Structure, 7, 133-139.

https://doi.org/10.1016/j.molstruc.2013.12.057

[15] Wu, X.X., Wang, G.J., Zhu, X.D., Huang, J. and Lv, J.Y. (2014) Tracking Analysis of Octacosanol in the Extraction of Arenaria polytrichoide by Fourier Transform Infrared Spectroscopy. Journal of Yunnan Nationalities University (Natural Science Edition), 1, 24-27.

[16] Lu, L.B., Wu, X.X., Yang, S., Shi, Y.J. and Yu, K. (2013) Analysis of Saccharum officinarum Bark and Its Extracts by Fourier Transform Infrared Spectroscopy. Journal of Yuxi Normal University, 12, 13-16. 